\title{
Urinary kallikrein 10 predicts the incurability of gastric cancer
}

\author{
Takaya Shimura ${ }^{1}$, Masahide Ebi ${ }^{1,2}$, Tomonori Yamada ${ }^{3}$, Tamaki Yamada ${ }^{4}$, Takahito \\ Katano1, Yu Nojiri ${ }^{1,3}$, Hiroyasu Iwasaki ${ }^{1,3}$, Satoshi Nomura ${ }^{1}$, Noriyuki Hayashi ${ }^{1}$, \\ Yoshinori Mori ${ }^{1}$, Hiromi Kataoka ${ }^{1}$, Marsha A. Moses ${ }^{5,6}$, Takashi Joh ${ }^{1}$ \\ ${ }^{1}$ Department of Gastroenterology and Metabolism, Nagoya City University Graduate School of Medical Sciences, Nagoya, \\ Japan \\ ${ }^{2}$ Department of Gastroenterology, Aichi Medical University, Nagakute, Japan \\ ${ }^{3}$ Department of Gastroenterology, Japanese Red Cross Nagoya Daini Hospital, Nagoya, Japan \\ ${ }^{4}$ Okazaki Public Health Center, Okazaki, Japan \\ ${ }^{5}$ Vascular Biology Program, Boston Children's Hospital, Boston, MA, USA \\ ${ }^{6}$ Department of Surgery, Harvard Medical School and Boston Children's Hospital, Boston, MA, USA \\ Correspondence to: Takaya Shimura, email: tshimura@med.nagoya-cu.ac.jp
}

Keywords: biomarker, gastric cancer, inoperability, kallikrein 10, urine

Received: November 08, $2016 \quad$ Accepted: February 22, $2017 \quad$ Published: March 22, 2017

Copyright: Shimura et al. This is an open-access article distributed under the terms of the Creative Commons Attribution License (CC-BY), which permits unrestricted use, distribution, and reproduction in any medium, provided the original author and source are credited.

\section{ABSTRACT}

The current imaging modalities are not sufficient to identify inoperable tumor factors, including distant metastasis and local invasion. Hence, we conducted this study using urine samples to discover non-invasive biomarkers for the incurability of gastric cancer (GC). Urine samples from 111 GC patients were analyzed in this study. The GC cohort was categorized and analyzed according to disease stage and operability. In the discovery phase, protease protein array analysis identified 3 potential candidate proteins that were elevated in the urine of advanced GC patients compared to early GC patients. Among them, urinary kallikrein 10 (KLK10) was positively associated with tumor stage progression. Moreover, the urinary level of KLK10 (uKLK10) was significantly elevated in the urine of patients with inoperable GC compared to operable GC patients (median, 118 vs. 229; $P=0.014$ ). The combination of uKLK10, tumor location and tumor size distinguished operability of GC with an area under the curve of $0.859,82.4 \%$ sensitivity and $86.2 \%$ specificity. Disease-free survival (DFS) was significantly shorter in GC patients with high uKLK10 compared to those with low uKLK10 (hazard ratio: 3.30 [95\% confidence interval, 1.58-6.90] $P<0.001)$. Immunohistochemical analyses also demonstrated a positive correlation between tumor stage and KLK10 expression in GC tissues $(r=0.426, P<0.001)$. In addition, GC patients with high expression of pathological KLK10 (pKLK10) showed a significantly shorter DFS compared to those with low pKLK10 (hazard ratio: 3.79 [95\% confidence interval, $1.27-11.24] P=0.010)$. uKLK10 is a promising non-invasive biomarker for the inoperability and incurability of GC.

\section{INTRODUCTION}

Gastric cancer (GC) is the fourth most common malignancy and the second leading cause of cancer deaths in the world [1]. The standard treatment is endoscopic and/ or surgical resection for operable stage I-III GC. However, surgical resection is not indicated for unresectable local advanced GC and metastatic GC, and neoadjuvant or palliative chemotherapy is systemically administered for these conditions. Preoperative staging diagnosis is thus very important for choosing an optimal treatment for GC. 
Contrast-enhanced computed tomography (CECT) has generally been used as a diagnostic tool for staging GC for operability. CECT can detect metastasis in the lymph nodes and in distant organs such as the liver and lungs, with tumor size and enhanced intratumor intensity. However, it is difficult to diagnose metastasis when the metastatic tumor size is small [2]. It has been reported that CECT sensitivity, specificity, and accuracy for lymph node metastasis (LNM) detection were 25-51\%, 79$92 \%$, and $64-72 \%$, respectively $[3,4]$. It is sometimes difficult to judge whether a tumor has invaded into an adjacent organ and is unresectable. Moreover, difficulty with the preoperative diagnosis of peritoneal metastasis is a considerable problem for GC treatment. In general, CECT may provide good accuracy for detecting liver metastasis, but does not have satisfactory diagnostic power for detecting pancreatic invasion, LNM, or peritoneal metastasis. CECT could not correctly diagnose GC in $45 \%$ of stage IV patients [5].

${ }^{18} \mathrm{~F}$-fluoro-2-deoxyglucose positron emission tomography (FDG-PET) has recently been developed to diagnose GC stage, and is usually integrated with computed tomography (FDG-PET/CT) to provide additional diagnostic power for malignancies [6]. However, FDG-PET/CT did not show additional diagnostic power for LNM compared to CECT where sensitivity, specificity, and accuracy of FDG-PET/CT vs. CECT were $34-41 \%$ vs. $51-75 \%$, $88-100 \%$ vs. $51-92 \%$, and $51-58 \%$ vs. $64-72 \%$, respectively $[3,4]$. FDG-PET/ CT also showed low sensitivity for peritoneal metastasis [7] and a high detectability of liver and lung metastasis [8], as did CECT. In a previous meta-analysis, sensitivity/ specificity for the detection of liver and peritoneal metastasis was, respectively, $54 \% / 98 \%$ and $9 \% / 99 \%$ on ultrasound, $74 \% / 99 \%$ and $33 \% / 99 \%$ on CT, and $70 \% / 96 \%$ and $28 \% / 97 \%$ on FDG-PET/CT [7]. Hence, the quality of current imaging modalities is not sufficient owing to the high false-negative rate. This results in patients with incurable or unresectable GC undergoing unnecessary and costly invasive surgery.

To overcome the limitations of the current imaging modalities, staging laparoscopy has been used to decide an optimal treatment for advanced GC. Staging laparoscopy could improve the diagnostic accuracy of metastasis from $37.8 \%$ with CT to $73 \%$, according to a previous study [9]. In particular, staging laparoscopy might be useful for the detection of peritoneal metastasis using direct observation, biopsy, and lavage cytology. Sensitivity, specificity, and accuracy of staging laparoscopy for peritoneal metastasis ranged from $73.7-100 \%, 83-100 \%$, and 93.4$100 \%$, respectively, in a previous systemic review [10]. However, it is occasionally difficult to detect peritoneal micrometastasis even if peritoneal lavage cytology is performed [11]. Moreover, staging laparoscopy is still invasive with consumption of cost and time.
The serum tumor markers CEA and CA19-9 have sometimes been used in clinical practice; however, they are ineffective for judging operability because of their low sensitivity, even for advanced stage GC (20-50\%) [12]. In fact, the latest meta-analysis, which analyzed results from over $5000 \mathrm{GC}$ patients in 46 studies, also reported that the sensitivity of CEA and CA19-9 was only $24.0 \%$ and $27.0 \%$ for all patients and $39.5 \%$ and $44.7 \%$ for patients with stage IV disease, respectively [13]. Non-invasive biomarkers that can predict the operability and curability of GC are therefore required. We herein demonstrate the usefulness of an urinary biomarker for detecting incurable GC.

\section{RESULTS}

\section{Patients}

The characteristics of the patients in the present study are shown in Table 1. In total, urine samples of 128 patients, 17 healthy controls and 111 with GC, were analyzed in this study. Among the 111 patients with GC, 94 patients had operable GC of stages I-III and 17 patients had inoperable GC of stages III and IV. Of 17 inoperable GC patients, 2 patients were locally advanced GCs and 15 patients had distant metastases which were observed in 7 at liver, 7 at lymph node, 2 at lung, 4 at peritoneum and 1 at bone. Of 94 operable GCs, only 1 did not undergo surgery due to severe co-morbidity, but the GC of the other 93 patients was successfully resected using endoscopy and surgery. No significant differences were noted for age, sex and serum creatinine level among healthy control, operable and inoperable GCs. In the inoperable GC group, the median primary tumor size was larger and the population included more cardia cancers compared to the operable GC group.

\section{Urinary protein array analyses}

We performed proteinase protein arrays to identify potential candidate biomarkers involved in GC progression, using 4 urine samples from 2 age- and sexmatched early GC patients and 2 advanced GC patients.

As shown in Figure 1, the expression levels of proteinase 3 (PRTN3), matrix metalloproteinase 9 (MMP9), and kallikrein 10 (KLK10) were increased in the urine of advanced GC patients, compared with the urine of early GC patients.

\section{Enzyme-linked immunosorbent assays}

Based on the results from the protease array analyses, we analyzed the urinary protein concentrations of 3 factors, PRTN3, MMP-9, and KLK10, in the whole cohort, using quantitative mono-specific ELISAs. 
Table 1: Characteristics of the study groups

\begin{tabular}{|c|c|c|c|c|c|c|}
\hline & & \multicolumn{2}{|c|}{ Gastric Cancer } & \multirow[b]{2}{*}{$P$} & \multirow{2}{*}{$\begin{array}{c}\text { Healthy control } \\
(\mathrm{N}=17)\end{array}$} & \multirow[b]{2}{*}{$P$} \\
\hline & & $\begin{array}{c}\text { Operable } \\
(\mathrm{N}=94)\end{array}$ & $\begin{array}{c}\text { Inoperable } \\
(\mathrm{N}=17)\end{array}$ & & & \\
\hline Age (years) & Median (IQR) & $67(62-73)$ & $72(65-76)$ & $0.340^{\# 1}$ & $68(63-72)$ & $0.626^{\# 4}$ \\
\hline \multirow[t]{2}{*}{ Sex } & Male & 74 & 12 & $0.529^{\# 2}$ & 12 & $0.631^{\# 3}$ \\
\hline & Female & 20 & 5 & & 5 & \\
\hline Serum Cr (mg/dl) & Median (IQR) & $0.80(0.70-0.90)$ & $0.73(0.60-1.10)$ & $0.967^{\# 1}$ & $0.88(0.67-0.94)$ & $0.906^{\# 4}$ \\
\hline Tumor size (mm) & Median (IQR) & $23(12-44)$ & $54(50-70)$ & $<0.001^{\# 1}$ & & \\
\hline \multirow[t]{2}{*}{ Histology, N (\%) } & Intestinal type & $70(74.5)$ & $10(58.8)$ & $0.240^{\# 2}$ & & \\
\hline & Diffuse type & $24(25.5)$ & $7(41.2)$ & & & \\
\hline \multirow[t]{2}{*}{ Location, N (\%) } & Cardia & $4(4.3)$ & $4(23.5)$ & $0.018^{\# 2}$ & & \\
\hline & Non-cardia & $90(95.7)$ & $13(76.5)$ & & & \\
\hline \multirow[t]{4}{*}{ Stage, N (\%) } & I & $71(75.5)$ & $0(0)$ & $<0.0011^{\# 3}$ & & \\
\hline & II & $12(12.8)$ & $0(0)$ & & & \\
\hline & III & $11(11.7)$ & $2(11.8)$ & & & \\
\hline & IV & $0(0)$ & $15(88.2)$ & & & \\
\hline \multirow[t]{3}{*}{ Resection, N (\%) } & Endoscopy & $48(51.1)$ & $0(0)$ & $<0.0011^{\# 3}$ & & \\
\hline & Gastrectomy & $45(47.9)$ & $1(5.9)$ & & & \\
\hline & Non-resection & $1(1.1)$ & $16(94.1)$ & & & \\
\hline
\end{tabular}

N, Number; Cr, Creatinine;

${ }^{\# 1}$, the Mann-Whitney U test; ${ }^{\# 2}$, Fisher's exact probability test; ${ }^{\# 3}$, the $\chi^{2}$ test; ${ }^{\# 4}$, Kruskal Wallis test.

Clinical stage is according to the seventh edition of UICC-TNM classification.

Urinary levels of all proteins were normalized to urinary creatinine $(\mathrm{uCr})$.

First, we analyzed urinary values of the 3 proteins according to disease stage. Urinary levels of PRTN3 (uPRTN3) and KLK10 (uKLK10) showed a significant positive correlation with disease stage, whereas the urinary level of MMP-9 (uMMP-9) did not (Figure 2A). Next, urinary protein levels were compared between the healthy control, operable GC, and inoperable GC groups. uKLK10 was significantly higher in the inoperable GC group compared to the operable GC group (healthy control vs. operable GC vs. inoperable GC: median uKLK10/uCr, 74 [interquartile range (IQR), 46-207] vs.118 [IQR, 56-235] vs. 229 [IQR, 105-450]), but uPRTN3 was not significant (Figure 2B). Hence, uKLK10 might be considered a biomarker of inoperable GC.

Moreover, uKLK10 significantly distinguished between the operable and inoperable GC groups in Receiver operating characteristic (ROC) curve analyses (Figure 3). When tumor location and size, which were significant factors in the inoperable GC groups shown in Table 1, were combined with uKLK10, it demonstrated a great accuracy in distinguishing between operable and inoperable GC (uKLK10: area under the curve $(\mathrm{AUC})=0.688[95 \%$ confidence interval $(\mathrm{CI}), 0.555$ 0.820], $P<0.014$; uKLK10+tumor location: $\mathrm{AUC}=0.768$ [95\% CI, 0.655-0.881], $P<0.001$; uKLK10+tumor location+size: $\mathrm{AUC}=0.859 \quad$ [95\% CI, 0.789-0.928], $P<0.001)$. When the cut-off values were determined as $>45 \mathrm{~mm}$ tumor size and $\geq 180$ in $\mathrm{uKLK} 10 / \mathrm{uCr}$ or cardia GC, inoperable GC could be detected with $82.4 \%$ sensitivity and $86.2 \%$ specificity.

Of 17 inoperable GC patients of this study, the inoperable tumor factors were found in 5 patients during surgery, which were not detected in the CT scan before surgery (sensitivity: 70.1\%). Interestingly, our established combination biomarker could categorize all 5 patients into the inoperable GC group.

\section{Immunohistochemistry}

Next, KLK10 expression in GC tissues was analyzed using immunohistochemistry for patients who underwent surgical resection of a primary tumor. Of the $111 \mathrm{GC}$ patients, 94 underwent surgical resection of primary GC. Immunoreactive KLK10 was not detected in most of the early stage GC tissues (Figure 4A, 4B), but it was strongly 
detected in the advanced GC tissues, and was particularly highly expressed at the invasive front (Figure 4C, 4D). Moreover, immunostaining scores for KLK10 were significantly associated with disease stage (median score, stage I: 0 [IQR, 0-2] vs. stage II: 4 [IQR, 3-5] vs. stage III: 5 [IQR, 3-6] vs. stage IV: 2 [IQR, 2-2]; $\mathrm{r}=0.426, P<0.001)$ (Figure 4E). Of the patients with stage IV GC, only 1 sample was available for the immunohistochemical study because most of them did not undergo primary resection.

\section{Disease-free survival}

According to urinary level and pathological expression of KLK10, disease-free survival (DFS) curves are shown in Figure 5A and 5B. When the GC cohort was categorized into 2 groups according to urinary levels of KLK10, the high uKLK10 group showed a significantly shorter DFS compared to the low uKLK10 group (1y-DFS, $63.8 \%$ vs. $84.4 \%$, respectively, $P<0.001$; hazard ratio (HR): 3.30 [95\% CI, 1.58-6.90]).

The GC cohort was also divided into 2 groups according to pathological KLK10 expression (pKLK10) in primary gastric tumor tissues: the low pKLK10 group had

A.

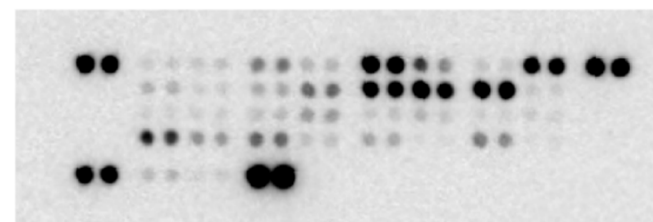

Early stage GC \#1

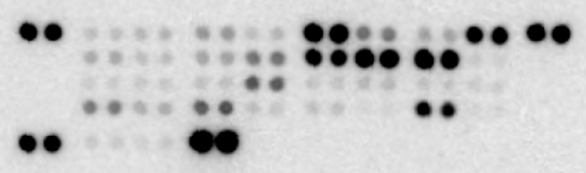

Early stage GC \#2

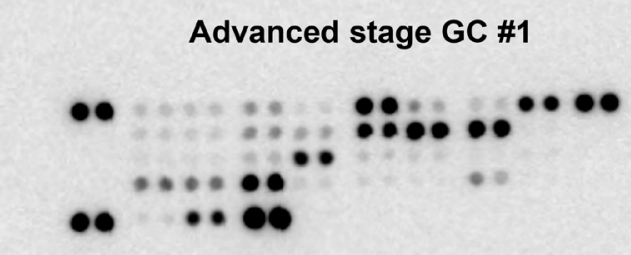

Advanced stage GC \#2

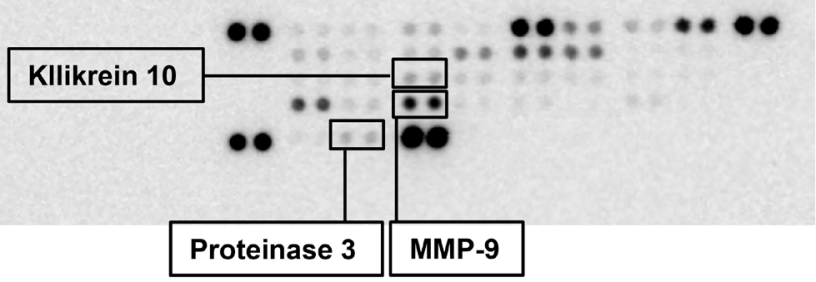

B.

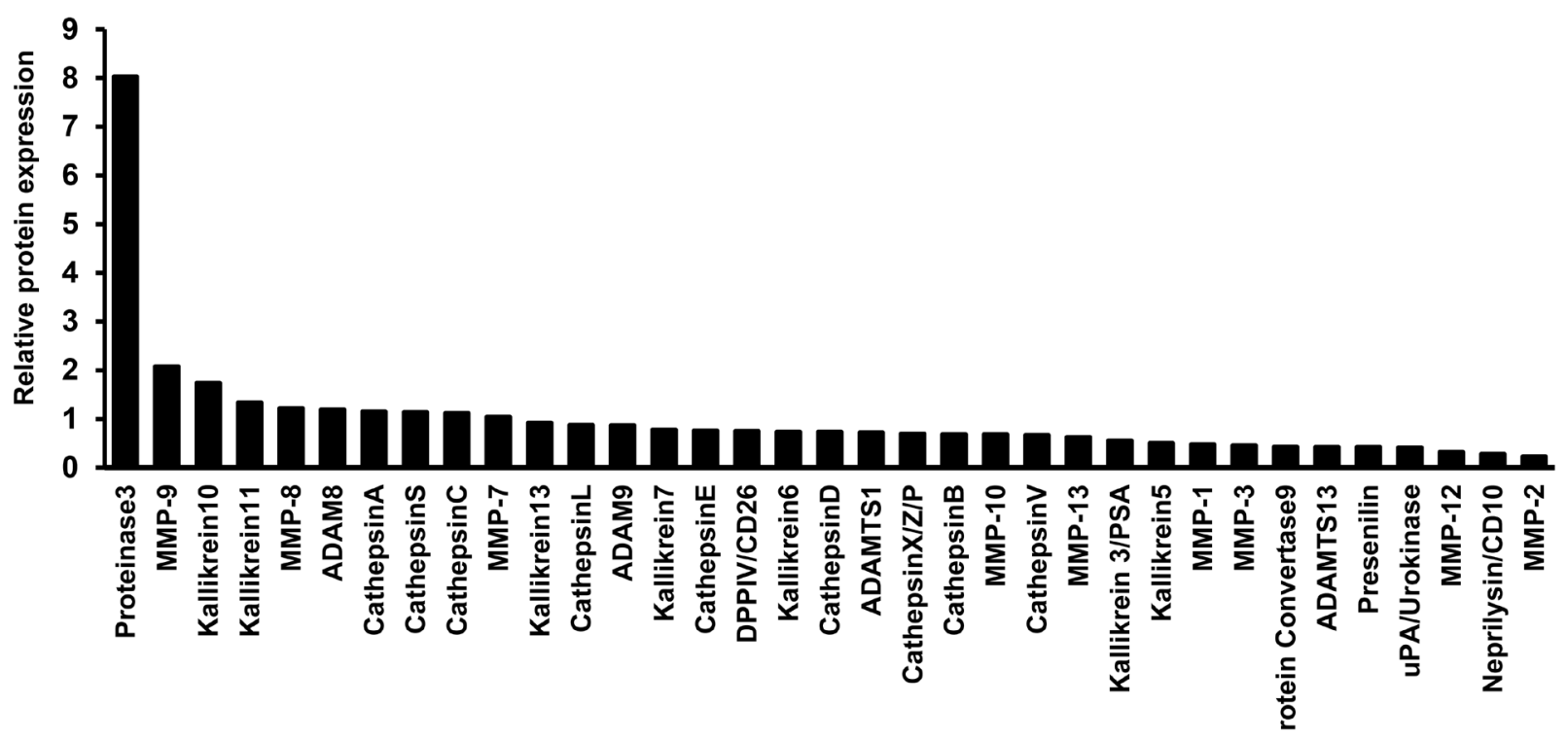

Figure 1: Protease array analyses of urine samples. (A) Urine samples from 2 patients with early gastric cancer (stage I) and 2 patients with advanced gastric cancer ( $\geq$ stage III), age- and sex-matched, were used in this analysis. (B) Quantification of relative protein expression by densitometry. Bar shows the mean relative protein expression value for 2 advanced gastric cancer patients, compared with 2 early gastric cancer patients. 
an immunostaining score of 0-2 while the high pKLK10 group had an immunostaining score of 3-7. The high pKLK10 group had significantly shorter DFS compared to the low pKLK10 group (1y-DFS, $89.3 \%$ vs. $92.6 \%$, respectively, $P=0.010$; HR: 3.79 [95\% CI, 1.27-11.24]).

\section{DISCUSSION}

In this study, using a variety of biochemical approaches, we have identified UKLK10 as a potential noninvasive biomarker of inoperable and incurable GC. Urine sampling is a straightforward and non-invasive procedure with low risks and costs, making it an attractive option for biomarker detection [14]. Moreover, urinary proteomics approach can reduce the masking effect [15] that obscures detection of low abundant proteins due to high abundant proteins. In fact, we have previously shown the usefulness of urinary biomarkers for the early detection of GC [16], as well as in the diagnosis of other malignancies [17-21].
It has also previously been shown that urinary biomarker protein levels are associated with disease progression in breast cancer [22]. Moreover, urinary biomarkers were useful for predicting the recurrence of some cancers after radiation therapy in another study [23]. Hence, urine is an important and non-invasive sample for the detection of cancer biomarkers.

The human tissue kallikrein and kallikrein-related peptidase (KLK) family has 15 members, making it the largest group of serine proteases [24]. KLKs are secreted proteins and some of those present in body fluids are considered attractive biomarkers for many cancers [24, 25]. The most successful biomarker KLK to date is serum KLK3, which is also called prostate-specific antigen (PSA): serum PSA has been widely used as a diagnostic biomarker for prostate cancer. KLK10, also known as normal epithelial cell-specific 1 (NES1), is a member of the KLK family with a molecular weight of $30 \mathrm{kDa}$ and was originally reported as a tumor suppressor in breast

A.
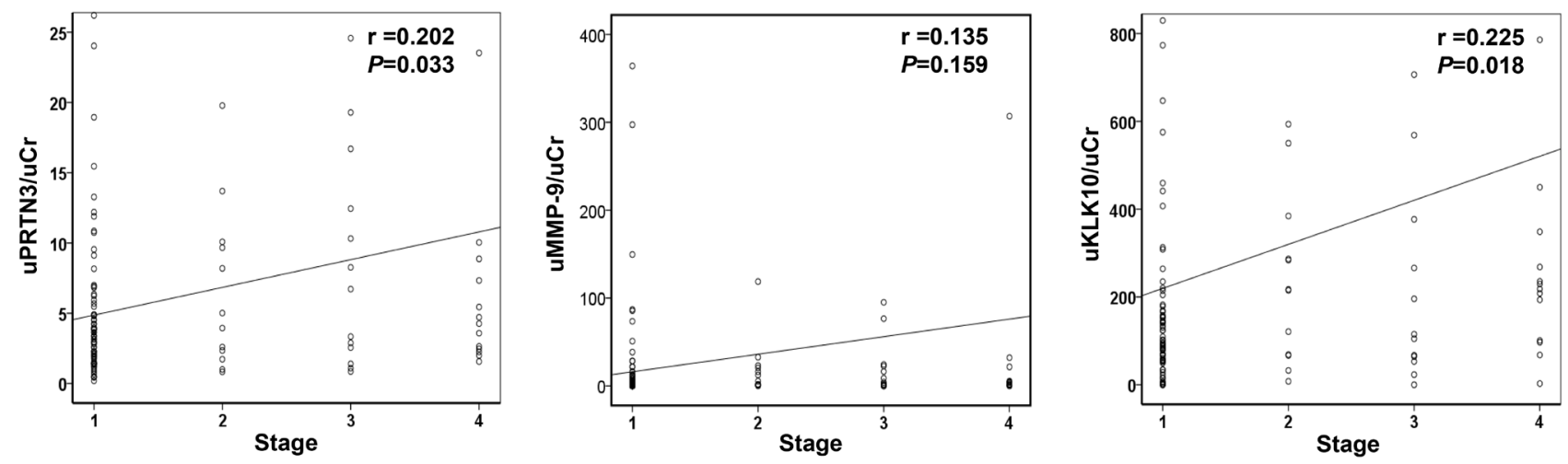

B.

$P=0.045$
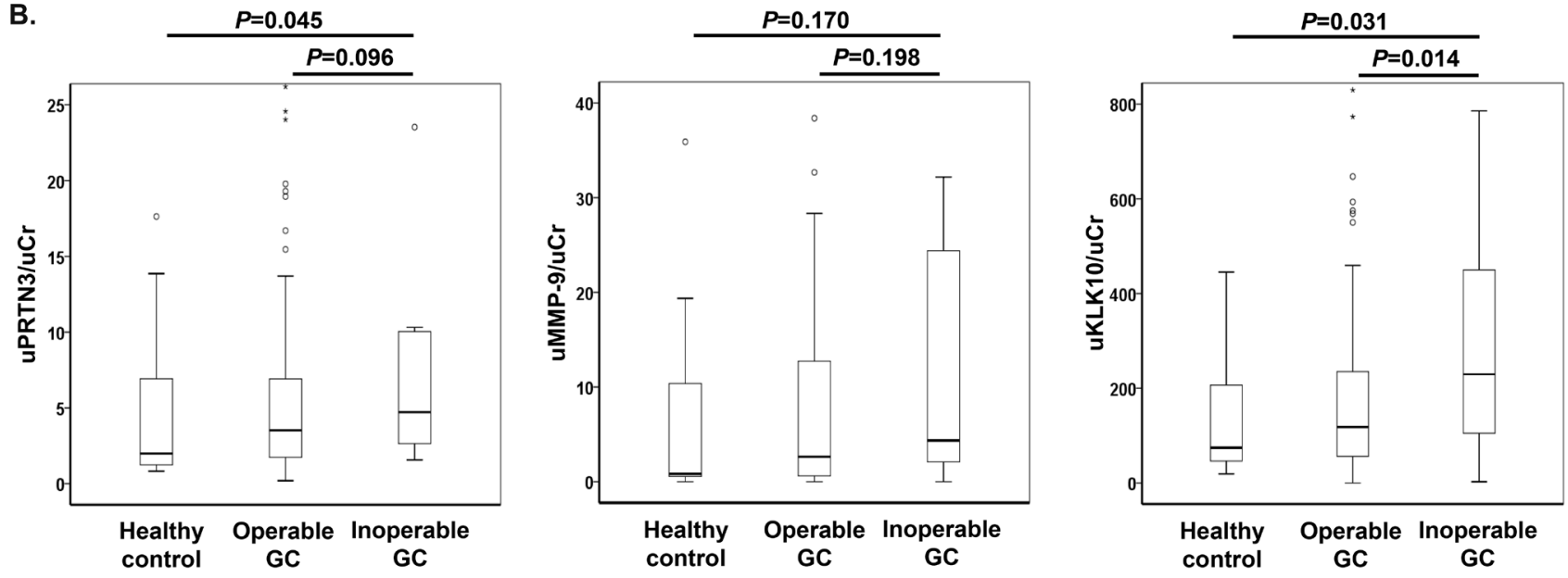

Figure 2: Urinary levels of proteinase 3, matrix metalloproteinase 9 and kallikrein-10. All urinary levels were normalized to urinary creatinine (uKLK10/uCr). (A) Correlation between urinary protein level and stage of gastric cancer. Data were analyzed using the Spearman rank correlation. (B) Urinary protein levels in healthy control, operable gastric cancer, and inoperable gastric cancer. Data were analyzed using the Mann-Whitney U test. PRTN3, proteinase 3; MMP-9, matrix metalloproteinase 9; KLK10, Kallikrein 10; GC, gastric cancer. 


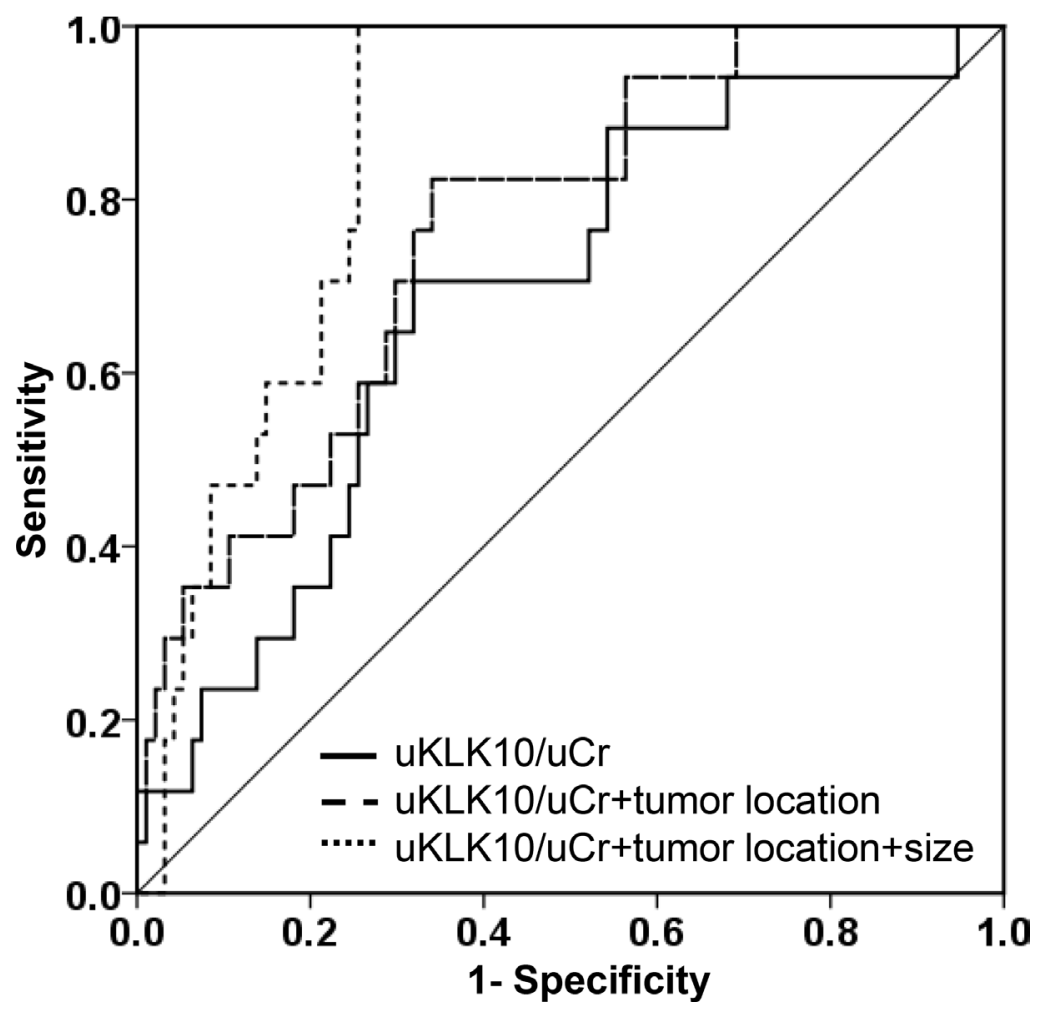

Figure 3: Receiver operating characteristic curves. Receiver operating characteristic curves were obtained from normalized urinary kallikrein 10 (uKLK10/uCr), tumor location and tumor size.
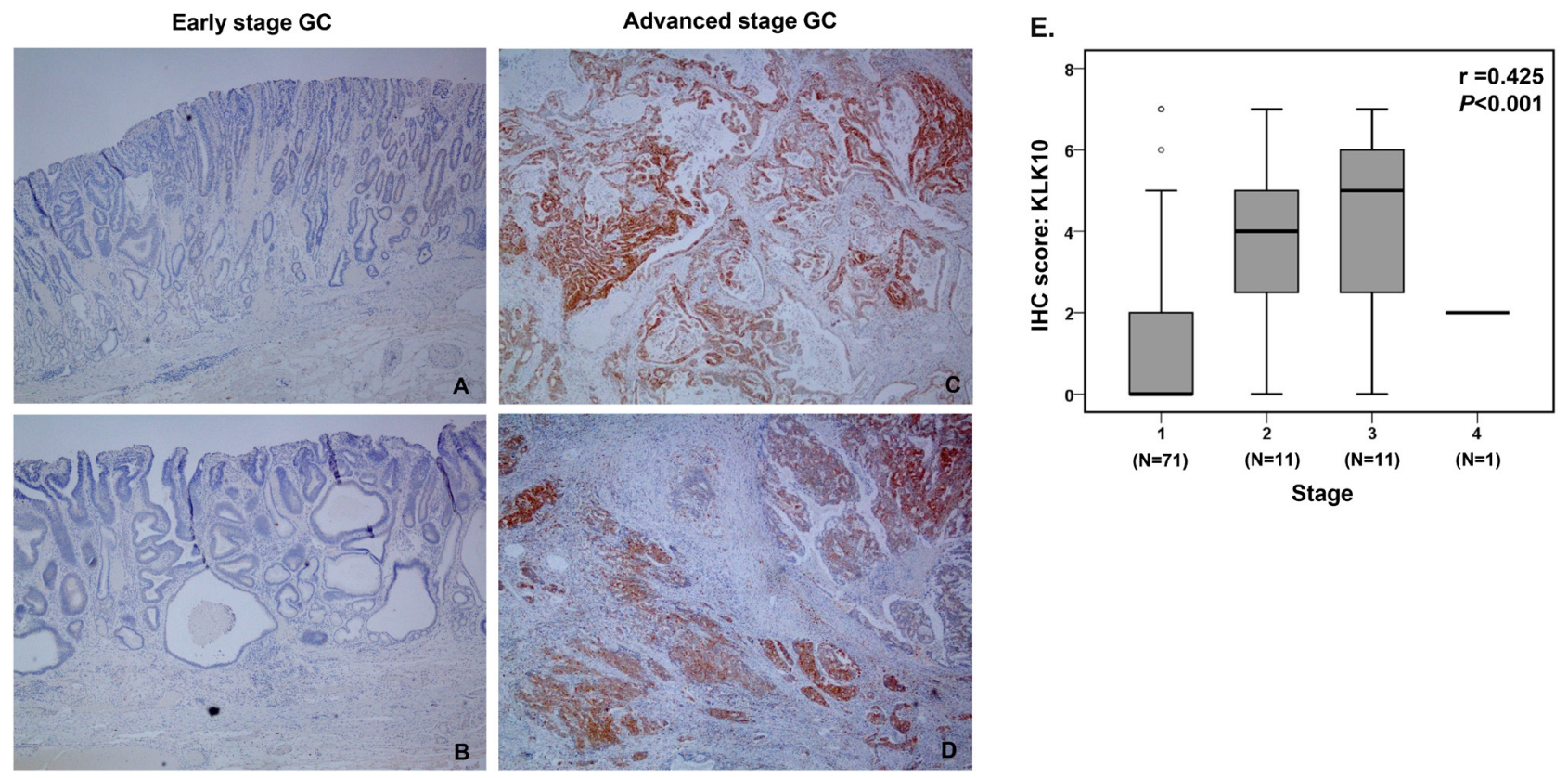

Figure 4: Immunohistochemical analyses of KLK10. (A-D) Representative images of KLK10 staining in early and advanced stage gastric cancers (x40). (A) No KLK10 expression was detected in early stage gastric cancer tissues (Stage IA, T1bN0M0). (B) No KLK10 expression was detected in other early stage gastric cancer tissues (Stage IA, T1aN0M0). (C) KLK10 was diffusely and strongly expressed in advanced stage gastric cancer tissues (Stage IIIA: T3N2M0). (D) KLK10 diffusely and strongly expressed in advanced stage gastric cancer tissues (Stage IIIC: T4aN3M0). (E) Quantitative analysis of immunohistochemistry. Staining scores were calculated in all gastric cancer surgical specimens and data were analyzed using the Spearman rank correlation. 
cancer cells [26]. In contrast, KLK10 promoted ovarian cancer cell growth in a recent study [27]. As for human studies, previous studies have demonstrated that mRNA expression of KLK10 was lower in breast cancer tissues [28], compared to normal tissues, whereas upregulation of KLK10 was associated with a poor prognosis or cancer diagnosis for other cancers including ovarian, pancreatic and colorectal cancers [29-31]. Hence, the true oncological significance and basic mechanism of action of KLK10 remains unclear. In terms of GC, there have been 8 studies that have reported KLK10 as a biomarker [32-39]. Most of these studies showed high expression of KLK10 in cancer lesions, although one study reported downregulation of KLK10 mRNA expression in GC tissues [37]. All previous reports utilized tissue samples, and analyzed protein and mRNA expression in tumor tissues. However, there have been no reports that detected the presence of circulating KLK10 in the urine and serum of GC patients.

Our study is the first demonstration of the presence of KLK10 in the urine of GC patients. In the present study, KLK10 levels were significantly higher in the urine of inoperable GC patients compared to operable GC patients, and they were positively associated with disease stage, as well as poor DFS. We also analyzed KLK10 protein expression in GC tissues following surgical resection. KLK10 expression in GC tissues was associated with advanced stage and poor DFS. Of the 8 previous studies described earlier in this Discussion, 5 reported that high expression of KLK10 in tumor tissue is associated with advanced stage or poor prognosis [32-35, 38], which supports our findings. In our study, DFS was significantly reduced in GC patients both high level of uKLK10 and pKLK10. This consistency of the result between uKLK10 and pKLK10 may suggest that urinary KLK10 might reflect tumoral expression of KLK10. Since KLK10 is

A.

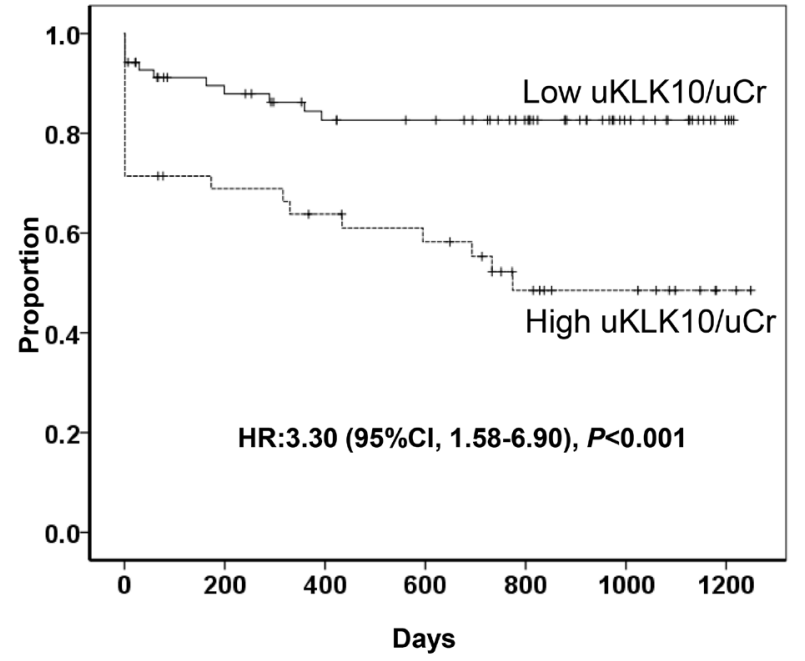

a secreted protein with low molecular weight, urinary measurement might reflect the dynamic KLK10 level in the whole body. Urine is easier and less invasive to collect than tumor tissue. Moreover, and importantly, assessment of pKLK10 would be difficult in biopsy specimens because KLK10 was expressed deep within the invasion margin. A previous study from Grin, et al. also has reported that KLK10 was expressed in the invasive front of GC [32]. Taken together, our use of urinary KLK10 as a biomarker has an advantage over using tumoral KLK10 expression.

There are some reports demonstrating that tumor size is a prognostic factor for GC [40-42], although size was not an independent prognostic factor in other studies $[43,44]$. Despite this controversy, tumor size might be a simple and useful parameter that can be easily obtained before treatment. Moreover, cardia GC also seems to be more advances stage and poorer prognosis compared to non-cardia GC $[45,46]$. The present study also demonstrated larger tumor size and more frequent cardia cancer in the inoperable GC group than in the operable GC group. The AUC obtained by multiplexing uKLK10, tumor location and size reached to an excellent level of 0.859 for predicting inoperable GC with $82.4 \%$ sensitivity and $86.2 \%$ specificity. Since our established biomarker correctly categorized 5 inoperable GC patients, who were not detected by CT, into the inoperable group, combination with CT findings may be also good strategy for predicting the inoperability of GC.

The current study has two following limitations. First, healthy controls of the present study were selected from a whole healthy control cohort using age- and sexmatching method. The previous studies have reported that KLK10 is synergistically regulated by steroid hormones such as estrogen, androgen, and progestin [47, 48], and this may influence uKLK10. Case-control method was

B.

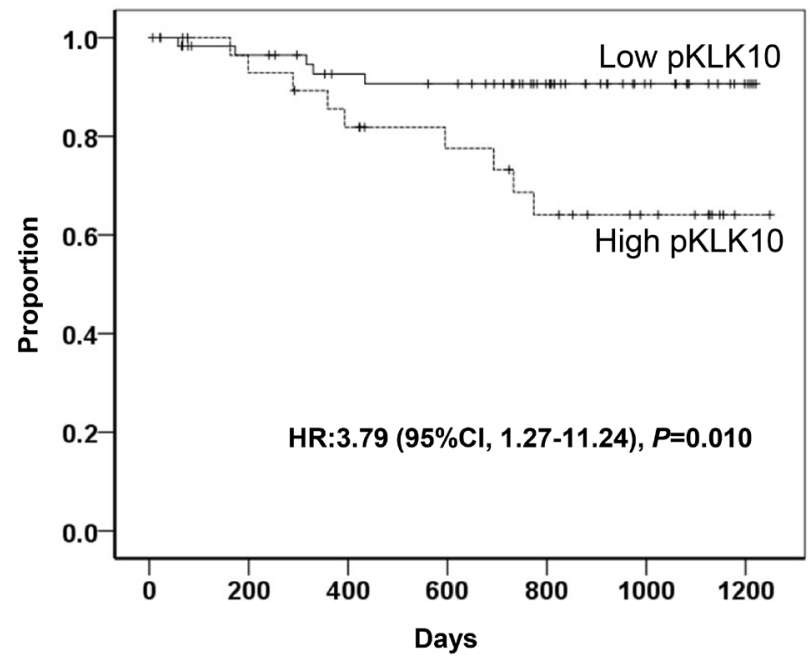

Figure 5: Disease-free survival. (A) Disease-free survival according to urinal level of KLK10. Urinary level of KLK10 was normalized to urinary creatinine (uKLK10/uCr). (B) Disease-free survival according to KLK10 expression in gastric cancer tissue. 
thus used for the healthy control to compensate the imbalances. Second, the operability was judged by the CT findings in some GCs without surgery, which may arise some biases. However, DFS data of the current study has also demonstrated that UKLK10 contributes to predicting relapse after surgery. Since it is impossible to detect micrometastasis with imaging modality, uKLK10 might be useful to predict the incurability as well as inoperability. In the future, it will be necessary to validate the efficacy of uKLK10 for future clinical applications in a prospective cohort study, as well as to determine oncological mechanisms of KLK10 in GC.

In conclusion, uKLK10 represents a non-invasive biomarker for predicting inoperable and incurable GC, which might help to identify optimal therapeutic and postoperative surveillance strategies.

\section{MATERIALS AND METHODS}

\section{Patients and study design}

All samples were prospectively collected from September 2012 to December 2015 at 3 participating Japanese institutions (Nagoya City University Hospital, Japanese Red Cross Nagoya Daini Hospital, and Okazaki Public Health Center). Patients who met all of the following inclusion criteria were enrolled in this study: aged between 20 and 90 years; histologically confirmed adenocarcinoma using biopsy for the GC group; no treatment before study enrollment for the GC group; and no neoplasms of any type for the healthy control group. Patients with a history of neoplasms of any type and/or with multiple neoplasms were excluded from enrollment in this study. Recurrent GC patients were excluded from this study. The present study included the healthy control cohort for whom age and sex were matched with inoperable GC patients one by one. Age difference within \pm 5 years was permitted when the sex was the same.

To assure the accuracy and comprehensiveness of reporting this case-control biomarker study, the present study complied with the REMARK guidelines [49] and the STROBE statement [50]. The study protocol was approved by the ethics committee at each institution and was conducted according to the ethical guidelines of the 1975 Declaration of Helsinki (6th revision, 2008). All patients provided written, informed consent before study entry. This study was registered with the University Hospital Medical Information Network Clinical Trials Registry (UMIN000021350).

\section{Samples and definition}

All urine samples were collected before any treatment for $\mathrm{GC}$, immediately frozen and stored at $-80^{\circ} \mathrm{C}$ until assayed, as previously reported [21]. Tissue samples of GC were obtained from primary tumors at the time of the initial surgical and endoscopic resection, fixed in formalin, and embedded in paraffin [51]. Clinical stage was determined by the final pathological diagnosis after resection, according to the 7 th edition of the Union for International Cancer Control (UICC) Tumor-NodeMetastasis (TNM) classification [52]. GC with stage IV or unresectable local invasion in the final diagnosis after surgery was defined as the inoperable GC. As for patients without surgery, when distant metastases and severe local invasion were clearly observed by CT scan, it was defined as the inoperable GC.

DFS was defined as the time from the date of cancer diagnosis until the first tumor appearance or death from any cause. In all patients, CT evaluations were performed every 3 to 6 months. The tumor size of GC was defined as the maximum size of the primary tumor on a CT scan at the time of diagnosis.

\section{Protein array analysis}

Since tissue degradation and adhesion by proteases are involved in cancer invasion and metastasis through the cleavage of relevant proteins, we hypothesized that proteases would be potentially useful biomarkers for advanced GC. To screen for urinary proteins in the urine of advanced GC patients, human protease arrays which contain 34 targets were utilized (R\&D Systems, Inc., Minneapolis, MN, USA), according to the manufacturer's instructions. We randomly selected 2 early GC patients (stage I) and 2 advanced GC patients ( $\geq$ stage III) who were completely matched by age and sex, and these 4 urine samples were incubated with antibody-coated array membranes at $4{ }^{\circ} \mathrm{C}$ overnight. To determine the relative expression level of proteins in advanced GC samples compared to early GC samples, the signal intensities of each protein were quantified by densitometry software, Image Quant TL (GE Healthcare Japan, Tokyo, Japan).

\section{Enzyme-linked immunosorbent assays}

We measured the urinary protein concentration of each of the proteins of interest using mono-specific enzyme-linked immunosorbent assays (ELISAs), according to the manufacturer's instructions. To measure each protein concentration, we used a Quantikine ELISA kit (R\&D Systems, Inc.) for MMP-9, a DuoSet ELISA with the DuoSet Ancillary Reagent Kit 2 (R\&D Systems, Inc.) for PRTN3, a human kallikrein 10 ELISA kit (RayBiotech, Inc., Norcross, GA, USA) for KLK10, and a Parameter Creatinine Assay (R\&D Systems, Inc.) for creatinine. All urine samples were measured in duplicate, and the mean was obtained. 


\section{Immunohistochemistry}

Immunohistochemical staining was performed using serial sections of each sample as follows. Consecutive sections ( $5 \mu \mathrm{m}$ thick) were deparaffinized and dehydrated through a graded series of xylene and ethanol. After inhibiting endogenous peroxidase activity with 3\% hydrogen peroxide (DAKO, Carpinteria, CA, USA) for 5 minutes, blocking of nonspecific binding sites was performed using Protein Block Serum-Free (DAKO) for 20 minutes, according to the manufacturer's instruction. Each section was incubated with a rabbit anti-human KLK10 primary antibody (\#bs-2531R: $5 \mu \mathrm{g} / \mathrm{ml}$, Bioss Inc., Woburn, MA, USA) at $4{ }^{\circ} \mathrm{C}$ overnight. Samples were then incubated with an anti-rabbit-horseradish peroxidase secondary antibody (DAKO), and immune complexes were visualized by incubation in 3,3'-diaminobenzidine (DAKO). Mayer's hematoxylin was used for nuclear counterstaining.

All immunostained specimens were assessed by an observer blinded to all clinical information. The staining intensity was classified as negative (0), weak $(1+)$, moderate $(2+)$, or strong $(3+)$, and the extent of the staining was defined as the percentage of positive staining areas scored on a scale of $0-4$ as follows: $0,0 \% ; 1,1-25 \%$; $2,26-50 \% ; 3,51-75 \% ; 4,76-100 \%$, as described in a previous study [53]. The sum of the staining intensity and staining extent scores was used as the final staining score.

\section{Statistical analyses}

The aim of this study was to identify urinary proteins that can diagnose the operability of GC. Quantitative variables were described with the median and IQR and analyzed using the Mann-Whitney $U$ test. Other data were analyzed using the $\chi^{2}$ test or Fisher's exact probability test, as appropriate. The nonparametric Spearman's rank correlation coefficient (r) was used as a measure of correlation. KaplanMeier curves were constructed in order to analyze DFS, and differences between the two groups were compared with a log-rank test. Cox proportional hazards model was used to calculate each HR.

The ROC analysis was used to calculate the AUC for each biomarker, and the representative value was shown as the AUC value with $95 \% \mathrm{CI}$. The true-positive fractions and the false-positive fractions for diagnosis of GC were calculated for every cut-off level, and the cutoff level was determined by selecting the farthest point from the baseline using ROC analysis. For combinations of biomarkers, the estimated coefficients of a logistic regression model were used to construct a composite score, which was then used to calculate the AUC for the combination of biomarkers. A two-tailed $P$ value of less than 0.05 was considered statistically significant.

\section{ACKNOWLEDGMENTS}

We thank Yukimi Hashidume-Itoh for handling of urine sample and Takako Onodera for data management of enrolled patients in this study (Department of Gastroenterology and Metabolism, Nagoya City University Graduate School of Medical Sciences). We are also grateful to our clinical colleagues who assisted in the sample collection.

\section{CONFLICTS OF INTEREST} study.

All authors have no conflicts of interest for this

\section{GRANT SUPPORT}

This research was supported by the Aichi Cancer Research Foundation (to T. Shimura) and the Advanced Medical Research Foundation (to M.A. Moses).

\section{REFERENCES}

1. Ferlay J, Shin HR, Bray F, Forman D, Mathers C, Parkin DM. Estimates of worldwide burden of cancer in 2008: GLOBOCAN 2008. Int J Cancer. 2010; 127: 2893-917.

2. Fukuya T, Honda H, Hayashi T, Kaneko K, Tateshi Y, Ro T, Maehara Y, Tanaka M, Tsuneyoshi M, Masuda K. Lymphnode metastases: efficacy for detection with helical CT in patients with gastric cancer. Radiology. 1995; 197: 705-11.

3. Park K, Jang G, Baek S, Song H. Usefulness of combined $\mathrm{PET} / \mathrm{CT}$ to assess regional lymph node involvement in gastric cancer. Tumori. 2014; 100: 201-6.

4. Kim EY, Lee WJ, Choi D, Lee SJ, Choi JY, Kim BT, Kim HS. The value of PET/CT for preoperative staging of advanced gastric cancer: comparison with contrastenhanced CT. Eur J Radiol. 2011; 79: 183-8.

5. Adachi Y, Sakino I, Matsumata T, Iso Y, Yoh R, Kitano $\mathrm{S}$, Okudaira Y. Preoperative assessment of advanced gastric carcinoma using computed tomography. Am J Gastroenterol. 1997; 92: 872-5.

6. Bar-Shalom R, Yefremov N, Guralnik L, Gaitini D, Frenkel A, Kuten A, Altman H, Keidar Z, Israel O. Clinical performance of PET/CT in evaluation of cancer: additional value for diagnostic imaging and patient management. $\mathrm{J}$ Nucl Med. 2003; 44: 1200-9.

7. Wang Z, Chen JQ. Imaging in assessing hepatic and peritoneal metastases of gastric cancer: a systematic review. BMC Gastroenterol. 2011; 11: 19.

8. Chung HW, Lee EJ, Cho YH, Yoon SY, So Y, Kim SY, Lee MH, Kim JH, Lee SY, Sung IK, Park HS, Yoo MW, Lee KY. High FDG uptake in PET/CT predicts worse prognosis in patients with metastatic gastric adenocarcinoma. J Cancer Res Clin Oncol. 2010; 136: 1929-35. 
9. Blackshaw GR, Barry JD, Edwards P, Allison MC, Thomas GV, Lewis WG. Laparoscopy significantly improves the perceived preoperative stage of gastric cancer. Gastric Cancer. 2003; 6: 225-9.

10. Leake PA, Cardoso R, Seevaratnam R, Lourenco L, Helyer L, Mahar A, Law C, Coburn NG. A systematic review of the accuracy and indications for diagnostic laparoscopy prior to curative-intent resection of gastric cancer. Gastric Cancer. 2012; 15: S38-47.

11. Abe S, Yoshimura H, Tabara H, Tachibana M, Monden $\mathrm{N}$, Nakamura T, Nagaoka S. Curative resection of gastric cancer: limitation of peritoneal lavage cytology in predicting the outcome. J Surg Oncol. 1995; 59: 226-9.

12. Sturgeon CM, Duffy MJ, Hofmann BR, Lamerz R, Fritsche HA, Gaarenstroom K, Bonfrer J, Ecke TH, Grossman HB, Hayes P, Hoffmann RT, Lerner SP, Lohe F, et al. National Academy of Clinical Biochemistry Laboratory Medicine Practice Guidelines for use of tumor markers in liver, bladder, cervical, and gastric cancers. Clin Chem. 2010; 56: e1-48.

13. Shimada H, Noie T, Ohashi M, Oba K, Takahashi Y. Clinical significance of serum tumor markers for gastric cancer: a systematic review of literature by the Task Force of the Japanese Gastric Cancer Association. Gastric cancer. 2014; 17: 26-33.

14. Yang J, Roy R, Jedinak A, Moses MA. Mining the Human Proteome: Biomarker Discovery for Human Cancer and Metastases. Cancer J. 2015; 21: 327-36.

15. Di Meo A, Pasic MD, Yousef GM. Proteomics and peptidomics: moving toward precision medicine in urological malignancies. Oncotarget. 2016; 7: 52460-74. doi: 10.18632/oncotarget.8931.

16. Shimura T, Dagher A, Sachdev M, Ebi M, Yamada T, Yamada T, Joh T, Moses MA. Urinary ADAM12 and MMP-9/NGAL complex detect the presence of gastric cancer. Cancer Prev Res (Phila). 2015; 8: 240-8.

17. Roy R, Zurakowski D, Wischhusen J, Frauenhoffer C, Hooshmand S, Kulke M, Moses MA. Urinary TIMP-1 and MMP-2 levels detect the presence of pancreatic malignancies. Br J Cancer. 2014; 111: 1772-9.

18. Coticchia CM, Curatolo AS, Zurakowski D, Yang J, Daniels KE, Matulonis UA, Moses MA. Urinary MMP-2 and MMP-9 predict the presence of ovarian cancer in women with normal CA125 levels. Gynecol Oncol. 2011; 123: 295-300.

19. Smith ER, Zurakowski D, Saad A, Scott RM, Moses MA. Urinary biomarkers predict brain tumor presence and response to therapy. Clin Cancer Res. 2008; 14: 2378-86.

20. Fernandez CA, Yan L, Louis G, Yang J, Kutok JL, Moses MA. The matrix metalloproteinase-9/neutrophil gelatinaseassociated lipocalin complex plays a role in breast tumor growth and is present in the urine of breast cancer patients. Clin Cancer Res. 2005; 11: 5390-5.

21. Moses MA, Wiederschain D, Loughlin KR, Zurakowski D, Lamb CC, Freeman MR. Increased incidence of matrix metalloproteinases in urine of cancer patients. Cancer Res. 1998; 58: 1395-9.

22. Roy R, Wewer UM, Zurakowski D, Pories SE, Moses MA. ADAM 12 cleaves extracellular matrix proteins and correlates with cancer status and stage. J Biol Chem. 2004; 279: 51323-30.

23. Chan LW, Moses MA, Goley E, Sproull M, Muanza T, Coleman CN, Figg WD, Albert PS, Menard C, Camphausen $\mathrm{K}$. Urinary VEGF and MMP levels as predictive markers of 1 -year progression-free survival in cancer patients treated with radiation therapy: a longitudinal study of protein kinetics throughout tumor progression and therapy. J Clin Oncol. 2004; 22: 499-506.

24. Kontos CK, Scorilas A. Kallikrein-related peptidases (KLKs): a gene family of novel cancer biomarkers. Clin Chem Lab Med. 2012; 50: 1877-91.

25. Avgeris M, Mavridis K, Scorilas A. Kallikrein-related peptidase genes as promising biomarkers for prognosis and monitoring of human malignancies. Biol Chem. 2010; 391: 505-11.

26. Liu XL, Wazer DE, Watanabe K, Band V. Identification of a novel serine protease-like gene, the expression of which is down-regulated during breast cancer progression. Cancer Res. 1996; 56: 3371-9.

27. White NM, Chow TF, Mejia-Guerrero S, Diamandis M, Rofael Y, Faragalla H, Mankaruous M, Gabril M, Girgis A, Yousef GM. Three dysregulated miRNAs control kallikrein 10 expression and cell proliferation in ovarian cancer. $\mathrm{Br} \mathrm{J}$ Cancer. 2010; 102: 1244-53.

28. Dhar S, Bhargava R, Yunes M, Li B, Goyal J, Naber SP, Wazer DE, Band V. Analysis of normal epithelial cell specific-1 (NES1)/kallikrein 10 mRNA expression by in situ hybridization, a novel marker for breast cancer. Clin Cancer Res. 2001; 7: 3393-8.

29. Dorn J, Harbeck N, Kates R, Gkazepis A, Scorilas A, Soosaipillai A, Diamandis E, Kiechle M, Schmalfeldt B, Schmitt M. Impact of expression differences of kallikreinrelated peptidases and of UPA and PAI-1 between primary tumor and omentum metastasis in advanced ovarian cancer. Ann Oncol. 2011; 22: 877-83.

30. Iacobuzio-Donahue CA, Ashfaq R, Maitra A, Adsay NV, Shen-Ong GL, Berg K, Hollingsworth MA, Cameron JL, Yeo CJ, Kern SE, Goggins M, Hruban RH. Highly expressed genes in pancreatic ductal adenocarcinomas: a comprehensive characterization and comparison of the transcription profiles obtained from three major technologies. Cancer Res. 2003; 63: 8614-22.

31. Talieri M, Alexopoulou DK, Scorilas A, Kypraios D, Arnogiannaki N, Devetzi M, Patsavela M, Xynopoulos D. Expression analysis and clinical evaluation of kallikreinrelated peptidase 10 (KLK10) in colorectal cancer. Tumour Biol. 2011; 32: 737-44.

32. Grin A, Samaan S, Tripathi M, Rotondo F, Kovacs K, Bassily MN, Yousef GM. Evaluation of human tissue kallikrein-related peptidases 6 and 10 expression in early 
gastroesophageal adenocarcinoma. Hum Pathol. 2015; 46: 541-8.

33. Kolin DL, Sy K, Rotondo F, Bassily MN, Kovacs K, Brezden-Masley C, Streutker CJ, Yousef GM. Prognostic significance of human tissue kallikrein-related peptidases 6 and 10 in gastric cancer. Biol Chem. 2014; 395: 1087-93.

34. Jiao X, Lu HJ, Zhai MM, Tan ZJ, Zhi HN, Liu XM, Liu $\mathrm{CH}$, Zhang DP. Overexpression of kallikrein gene 10 is a biomarker for predicting poor prognosis in gastric cancer. World J Gastroenterol. 2013; 19: 9425-31.

35. Lei KF, Liu BY, Zhang XQ, Jin XL, Guo Y, Ye M, Zhu ZG. Development of a survival prediction model for gastric cancer using serine proteases and their inhibitors. Exp Ther Med. 2012; 3: 109-16.

36. Li M, Zhao ZW, Zhang Y, Xin Y. Over-expression of Ephb4 is associated with carcinogenesis of gastric cancer. Dig Dis Sci. 2011; 56: 698-706.

37. Huang W, Zhong J, Wu LY, Yu LF, Tian XL, Zhang YF, $\mathrm{Li}$ B. Downregulation and $\mathrm{CpG}$ island hypermethylation of NES1/hK10 gene in the pathogenesis of human gastric cancer. Cancer Lett. 2007; 251: 78-85.

38. Feng B, Xu WB, Zheng MH, Ma JJ, Cai Q, Zhang Y, Ji J, Lu AG, Qu Y, Li JW, Wang ML, Hu WG, Liu BY, et al. Clinical significance of human kallikrein 10 gene expression in colorectal cancer and gastric cancer. J Gastroenterol Hepatol. 2006; 21: 1596-603.

39. Yousef GM, White NM, Michael IP, Cho JC, Robb JD, Kurlender L, Khan S, Diamandis EP. Identification of new splice variants and differential expression of the human kallikrein 10 gene, a candidate cancer biomarker. Tumour Biol. 2005; 26: 227-35.

40. Zu H, Wang F, Ma Y, Xue Y. Stage-stratified analysis of prognostic significance of tumor size in patients with gastric cancer. PLoS One. 2013; 8: e54502.

41. Giuliani A, Caporale A, Di Bari M, Demoro M, Gozzo P, Corona M, Miccini M, Ricciardulli T, Tocchi A. Maximum gastric cancer diameter as a prognostic indicator: univariate and multivariate analysis. J Exp Clin Cancer Res. 2003; 22: 531-8.

42. Adachi Y, Oshiro T, Mori M, Maehara Y, Sugimachi K. Tumor size as a simple prognostic indicator for gastric carcinoma. Ann Surg Oncol. 1997; 4: 137-40.

43. Yokota T, Ishiyama S, Saito T, Teshima S, Narushima Y, Murata K, Iwamoto K, Yashima R, Yamauchi H, Kikuchi S. Lymph node metastasis as a significant prognostic factor in gastric cancer: a multiple logistic regression analysis. Scand J Gastroenterol. 2004; 39: 380-4.

44. Yokota T, Ishiyama S, Saito T, Teshima S, Yamada Y, Iwamoto K, Takahashi M, Murata K, Yamauchi H. Is tumor size a prognostic indicator for gastric carcinoma? Anticancer Res. 2002; 22: 3673-7.

45. Saito H, Fukumoto Y, Osaki T, Fukuda K, Tatebe S, Tsujitani S, Ikeguchi M. Distinct recurrence pattern and outcome of adenocarcinoma of the gastric cardia in comparison with carcinoma of other regions of the stomach. World J Surg. 2006; 30: 1864-9.

46. Bruno L, Nesi G, Montinaro F, Carassale G, Lassig R, Boddi V, Bechi P, Cortesini C. Clinicopathologic findings and results of surgical treatment in cardiac adenocarcinoma. J Surg Oncol. 2000; 74: 33-5.

47. Paliouras M, Diamandis EP. Androgens act synergistically to enhance estrogen-induced upregulation of human tissue kallikreins 10, 11, and 14 in breast cancer cells via a membrane bound androgen receptor. Mol Oncol. 2008; 1 : 413-24.

48. Luo LY, Grass L, Diamandis EP. Steroid hormone regulation of the human kallikrein 10 (KLK10) gene in cancer cell lines and functional characterization of the KLK10 gene promoter. Clin Chim Acta. 2003; 337: 115-26.

49. McShane LM, Altman DG, Sauerbrei W, Taube SE, Gion M, Clark GM. Reporting recommendations for tumor marker prognostic studies. J Clin Oncol. 2005; 23: 9067-72.

50. Vandenbroucke JP, von Elm E, Altman DG, Gotzsche PC, Mulrow CD, Pocock SJ, Poole C, Schlesselman JJ, Egger M. Strengthening the Reporting of Observational Studies in Epidemiology (STROBE): explanation and elaboration. Epidemiology. 2007; 18: 805-35.

51. Shimura T, Sasaki M, Kataoka H, Tanida S, Oshima T, Ogasawara N, Wada T, Kubota E, Yamada T, Mori Y, Fujita F, Nakao H, Ohara H, et al. Advantages of endoscopic submucosal dissection over conventional endoscopic mucosal resection. J Gastroenterol Hepatol. 2007; 22: 821-6.

52. Sobin LH, Gospodarowicz MK, Wittekind C. TNM classification of malignant tumours, 7th ed. Hoboken, NJ: John Wiley \& Sons, Inc;. 2009.

53. Masunaga R, Kohno H, Dhar DK, Ohno S, Shibakita M, Kinugasa S, Yoshimura H, Tachibana M, Kubota H, Nagasue N. Cyclooxygenase-2 expression correlates with tumor neovascularization and prognosis in human colorectal carcinoma patients. Clin Cancer Res. 2000; 6: 4064-8. 